\title{
Estimación de costos para la producción de guayaba rosada (Psidium guajava L.) en el sur de Florida ${ }^{1}$
}

\author{
Edward Evans, Fredy H. Ballen, Jonathan Crane, Trent Blare, Victor Contreras y Aditya Singh²
}

\section{Resumen}

La guayaba se cultiva alrededor del mundo en zonas subtropicales y tropicales ya que se adapta a una gran variedad de condiciones climáticas (de húmedas a secas). La Florida es el principal productor de guayaba en los EE.UU. este cultivo está valorado en $\$ 3.20$ millones a nivel de empacadora. En los últimos años ha crecido el interés en los cultivos tropicales alternos, este articulo tiene como objetivo servir como referencia para estimar los requerimientos financieros de una plantación establecida de guayaba en el sur de Florida. En este análisis estimamos que una plantación establecida de guayaba rosada tiene un retorno neto de \$9,065/acre/año (\$1.13/libra).

\section{Palabras clave}

frutas tropicales, mercados nichos, productos alternativos, presupuesto de producción

\section{Introducción}

La guayaba (Psidium guajava L.) es un miembro de la familia de las mirtáceas, es un árbol pequeño nativo del trópico americano, de uno o más troncos cuya hoja es perenne. La fruta presenta diferentes tamaños y formas; su sabor puede variar entre ácido y dulce. Esta puede presentar un aroma fuerte o suave, así como poseer una piel gruesa o fina según la variedad (Morton 1987; Mossler and Crane 2009).

La guayaba se cultiva alrededor del mundo en zonas subtropicales y tropicales ya que se adapta a una gran variedad de condiciones climáticas (de húmedas a secas). A nivel mundial, India y México son los principales productores (FAOSTAT 2016). México es el principal exportador de guayaba fresca a los Estados Unidos, abasteciendo de fruta durante todo el año a las principales ciudades (USDA-AMS 2017).

La guayaba fue introducida a Florida de Cuba en 1847 y rápidamente se naturalizo en las regiones centro y sur (Popenoe 1920). La producción de guayaba de EE. UU se concentra principalmente en Hawái, Puerto Rico y Florida (Crane and Balerdi 2016). La fruta se produce en menor escala en California y Texas; en Florida el área de cultivo de guayaba es aproximadamente 200 acres, alrededor de un 95\% del área se ubica en el condado de Miami-Dade.

Hay 2 tipos de guayaba cultivadas comercialmente en Florida, la rosada y la blanca (estas se caracterizan por el color de su pulpa o carne). Generalmente la guayaba rosada es consumida después de madurar mientras que la guayaba

1. This publication is FE1113, one of a series of the Food and Resource Economics Department, UF/IFAS Extension, original publication date February 2022. It is the Spanish version of the publication FE1036, original in English available at https://edis.ifas.ufl.edu/publication/FE1036. Este artículo es una versión en español de la publicación FE1036, original en inglés, disponible en https://edis.ifas.ufl.edu/publication/FE1036.

2. Edward Evans; director del centro y profesor, Departamento de Economía de Alimentos y Recursos; Fredy H. Ballen, analista de gestión de datos II, Departamento de Economía de Alimentos y Recursos; Jonathan Crane, profesor y especialista en frutas tropicales, Departamento de Ciencias Hortícolas; Trent Blare, profesor asistente, Departamento de Economía de Alimentos y Recursos, Victor Contreras, investigador, Departamento de Economía de Alimentos y Recursos, y Aditya Singh, investigador, Departamento de Economía de Alimentos y Recursos; UF IFAS Centro de Investigación y Educación Tropical (TREC), Homestead, FL.

The Institute of Food and Agricultural Sciences (IFAS) is an Equal Opportunity Institution authorized to provide research, educational information and other services

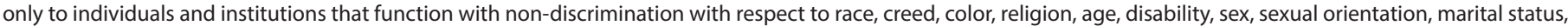

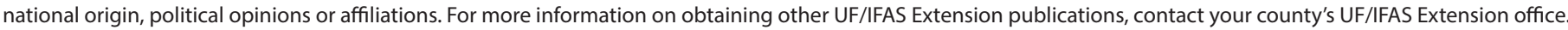
U.S. Department of Agriculture, UF/IFAS Extension Service, University of Florida, IFAS, Florida A \& M University Cooperative Extension Program, and Boards of County Commissioners Cooperating. Andra Johnson, dean for UF/IFAS Extension. 
blanca se consume en una etapa inmadura cuando ya está cerca de su tamaño óptimo (aun firme) (Crane and Balerdi 2016). Las principales variedades de guayaba rosada en Florida fueron seleccionadas por un productor local y están patentadas; el Centro Tropical de Investigación y Educación (TREC) de la Universidad de Florida desarrolló la variedad "Homestead" (Crane and Balerdi 2016). La temporada de cosecha principal de guayaba en Florida, es de agosto a octubre, seguida de una temporada pequeña de febrero a marzo (Mossler and Crane 2009). Los árboles de guayaba pueden ser inducidos a producir una cosecha fuera de temporada en cualquier época del año mediante manejo de podas, nutrientes y agua.

Un árbol de guayaba maduro (5 años o más) produce entre 50 a 80 libras de fruta por año dependiendo del tamaño, variedad, condiciones climáticas y prácticas culturales (Crane and Balerdi 2016). La guayaba rosada se siembra considerando una densidad de plantación de 150 árboles por acre; en promedio la producción de un árbol maduro es de 65 libras por año, con lo cual se calcula un rendimiento promedio de 9,750 libras/acre. Se estima que el rendimiento comercializable de guayaba rosada en Florida es de 82\%, aproximadamente 8,000 libras por acre; se estima que la producción comercializable de la Florida es de 1.6 millones de libras por año (200 acres X 8,000 libras/acre). Asumiendo un precio promedio "free on board" (FOB) en Homestead (es decir el precio local de la empacadora) de $\$ 2.00$ por libra, este cultivo está valorado en $\$ 3.20$ millones a nivel de empacadora.

Debido al creciente interés acerca de la rentabilidad de frutales tropicales alternativos, esta publicación presenta los costos y retornos asociados con la operación de una plantación ya establecida en el sur de Florida. La información presentada fue recolectada por medio de entrevistas con productores y expertos de la industria; esta refleja una gran variedad de técnicas de producción en fincas pequeñas de guayaba (1 a 3 acres). El propósito de la información presentada es servir como referencia para estimar los requerimientos financieros en la operación de una plantación ya establecida de guayaba rosada. Los lectores interesados (productores del gremio o personas interesadas en este cultivo) puede buscar información específica con respecto al cultivo de la guayaba pueden consultar HS4, Guava Growing in the Florida Home Landscape (https:// edis.ifas.ufl.edu/publication/MG045), o comunicarse con el agente UF/IFAS más cercano.

\section{Estimaciones principales}

El presupuesto anual y costos de producción están basados en una plantación de un acre. Debido a la gran diversidad de tipos de prácticas culturales adoptadas, solo los costos y retornos promedio son usados para el presente análisis. Debido a que los productores de frutas tropicales en el sur de Florida son dueños de la tierra, se consideró un costo de alquiler de $\$ 500 /$ acre por año como el costo de oportunidad de la tierra.

Plantación-la guayaba se planta de 20 a 25 pies entre filas y con una distancia de 10 a 20 pies entre plantas, lo que resulta en una densidad de plantación con rango aproximado de 87 a 217 árboles por acre (Mossler and Crane 2009). En el presente análisis se consideró una densidad de 150 árboles por acre.

Rendimiento-Se asume una densidad de plantación de 150 árboles/acre, un árbol maduro de guayaba produce en promedio 65 libras/año y un $82 \%$ de la fruta es comercializable, se estima una producción comercializable de 8,000 libras/acre/año.

Precio de venta-El precio FOB promedio (precio en la empacadora) de la guayaba rosada (en Homestad) se estima en $\$ 2.00 /$ libra, es el precio promedio que los productores recibieron de las empacadoras en el 2016.

Riego-Los árboles maduros de guayaba requieren una cantidad adecuada de riego durante los periodos de sequía para obtener un rendimiento óptimo, el riego también es crucial desde la floración hasta el desarrollo de la fruta (Crane and Balerdi 201). Los gastos promedios de riego incluyendo los costos de gasolina o electricidad se estiman en $\$ 200 /$ acre/año.

Fertilización-la fertilización de los árboles maduros incluyen aplicaciones de 6-6-6-2 o insumos similares (nitrógeno, fosfato, potasa y magnesio); aplicaciones dirigidas al suelo (drench) de quelato de hierro, así como aplicaciones foliares (zinc, manganeso, entre otros micronutrientes) (Crane and Balerdi 20216). Los costos de fertilización promedio (solo insumos) se estiman en $\$ 550 /$ acre/ año.

Manejo de plagas-Los árboles de guayaba se ven afectados por muchas plagas, entre las cuales, las de mayor importancia económica son la Mosca de la Fruta del Caribe (Anastrepha suspensa) y la Mosca Blanca de la Guayaba (Metaleurodicus cardini). 
Las prácticas de control de malezas incluyen la aplicación de herbicidas, deshierbe manual y uso de mulch. En el sur de Florida no hay enfermedades de gran impacto, sim embargo, hay incidencia de alga roja (Cephaleuros virescens) $\mathrm{y}$ antracnosis (Colletotrichum gloeosporioides), que pueden ser identificadas por la aparición de manchas rojas en las hojas (Crane and Balerdi 2016). El costo promedio de agroquímicos (solo insumos) es: insecticidas \$450/acre/año, herbicidas $\$ 450 /$ acre/año, y fungicidas $\$ 400 /$ acre/año.

Mano de obra-se incluyeron los costos de aplicación de insumos (fertilizantes y agroquímicos) y los costos de manejo de cultivo (riego, poda y otras). Los costos de mano de obra se estiman en \$650/acre/año.

Interés sobre capital-se refiere al costo del préstamo o el costo de oportunidad al usar el capital propio. Para este análisis se utilizó una tasa del 5\%, el interés sobre capital es de $\$ 135 /$ acre.

El cuadro 1 muestra los gastos y retornos por acre de una finca de guayaba rosada.

Costos de producción (o costos variables) los costos de producción se estiman en $\$ 2,835 /$ acre ( $\$ 0.35$ por libra), representando $40 \%$ de los costos totales. Los mayores componentes de los costos de producción son la mano de obra (23\%), fertilizantes (19\%), herbicidas (16\%) e insecticidas (16\%).

Costos fijos-se refiere a los costos independientes al nivel de producción. Estos incluyen los costos generales en efectivo $\$ 200$ (como seguro e impuestos), costos generales no en efectivo $\$ 500 /$ acre (costo de oportunidad de la tierra), y otros costos generales $\$ 800$ (uso de maquinaria, electricidad, teléfono, computadora, entre otros). El total de costos fijos se estima en $\$ 1,300 /$ acre/año ( $\$ 0.16 /$ libra), estos representan el $18 \%$ de los costos totales.

Cosecha y comercialización-La guayaba rosada generalmente se cosecha cuando la piel cambia de un color verde claro a uno amarillento (Crane and Balerdi 2016). Se asume que en la Florida se cosecha dos veces al año: una cosecha principal de agosto a octubre y una cosecha más pequeña de febrero a marzo, dependiendo de la variedad (Mossler and Crane 2009). Debido a que la fruta puede ser dañada fácilmente durante la cosecha, esta es cosechada a mano; la cosecha a mano es una actividad que consume gran cantidad de tiempo. Los costos de cosecha, empacado y mercadeo se estiman en $\$ 2,800 /$ acre/año ( $\$ 0.35 /$ libra), representando el $40 \%$ de los costos totales.
La figura 1 ilustra los porcentajes que representan los diferentes tipos de costos. En esta se puede observar que los costos de cosecha y comercialización en conjunto con los costos de producción (costos variables de mano de obra, riego, fertilización y control de plagas) son los principales, cada uno representa $40 \%$ de los costos totales; a estos le siguen costos fijos con $18 \%$ e interés sobre el capital con el $2 \%$.

\section{Estructura de Costos}

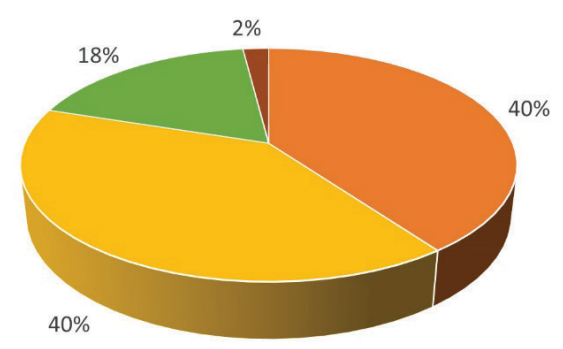

- Costos Culturales - Costos de Cosecha y Mercadeo - Costos Fijos - Interes Sobre Capital Figura 1. Proporción de Costos.

Credits: UF/IFAS

\section{Análisis de retorno y rentabilidad}

La información presentada en el cuadro 1 muestra que el costo total para producir y comercializar un acre de guayaba rosada es de $\$ 6,935 /$ acre/año ( $\$ 0.87 /$ libra). Si se estima un promedio de producción comercializable de 8,000 libras/acre y un precio promedio FOB en Homestead (en la empacadora) de $\$ 2.00 /$ libra, el ingreso total es de $\$ 16,000 /$ acre/año.

Al restar los costos variables (costos de producción más los costos de cosecha y comercialización) del ingreso total, se obtiene un margen bruto de $\$ 10,365 /$ acre/año ( $\$ 1.30 /$ libra). El margen bruto es un indicador de rentabilidad a corto plazo. Un margen bruto positivo indica que todos los costos variables han sido cubiertos por el ingreso total, mientras que un margen bruto negativo indica que una empresa o negocio no está cubriendo el total de sus costos operativos, cambios son necesarios para poder seguir operando.

Debido al hecho de que el margen bruto no incluye costos fijos, este no es un verdadero indicador de la viabilidad de un negocio o empresa a largo plazo.

El retorno neto se obtiene restando el costo fijo del margen bruto, este provee un indicador más real de una rentabilidad a largo plazo de una operación agrícola. El cuadro 1 muestra que una plantación de guayaba rosada tiene un retorno neto de $\$ 9,065 /$ acre/año ( $\$ 1.13 /$ libra). 


\section{Análisis de sensibilidad}

El cuadro 2 presenta un análisis basado en el margen bruto (ganancia bruta), en el cual se considera la rentabilidad de una plantación de guayaba rosada. En el mejor escenario en donde el precio y la producción se incrementan en un $10 \%$, el margen bruto por acre incrementa de $\$ 10,365 /$ acre a $\$ 13,725 /$ acre. En el peor escenario donde el precio y la producción caen un $10 \%$, el margen bruto por acre se reduciría de $\$ 10,365 /$ acre a $\$ 7,325 /$ acre. Si el precio cambia (manteniendo la producción constante) este tiene un impacto en el margen bruto similar a lo que pasaría si la producción cambia (manteniendo constante el precio). Se puede observar que un incremento de $5 \%$ en el precio base (con una producción de 8,000 libras) tiene un impacto en el margen bruto ( $\$ 800$ más) igual al impacto de un incremento en del $5 \%$ en la producción (precio base de $\$ 2.00 /$ libra). Hay que hacer énfasis en que el margen bruto estimado no incluye los costos fijos.

El cuadro 3 presenta un análisis similar basado en el retorno neto por acre. En el mejor escenario en donde el precio y la producción se incrementan un $10 \%$, el retorno neto por acre se incrementa de $\$ 9,065 /$ acre a $\$ 12,425 /$ acre. Bajo el peor escenario en donde el precio y la producción caen un $10 \%$, el retorno neto por acre se reduciría de $\$ 9,065 /$ acre a $\$ 6,025 /$ acre. Otras combinaciones de cambios en precio y/o producción y su impacto en el retorno neto se pueden observar en el cuadro 3, estas se interpretan de una manera similar a la presentada en el cuadro 2. Cabe hacer énfasis que, a un nivel industrial, un incremento significativo en la producción usualmente resulta en una caída de precios percibidos por los productores.

\section{Conclusiones}

El retorno promedio neto de una plantación establecida de guayaba rosada en el sur de Florida es $\$ 9,065 /$ acre (\$1.13/libra), lo cual vuelve a este cultivo una opción muy interesante en la región. La información presentada en este documento se basa en una plantación ya establecida; nosotros no hemos cuantificado los requisitos financieros para establecer una nueva plantación de guayaba, los cuales incluyen la adquisición de tierras, desarrollo, costos de siembra, amortización de capital, entre otros.

El panorama de la demanda de guayaba en EE. UU es favorable debido al incremento en la población étnica, particularmente la asiática e hispana que están familiarizados con la fruta. La sobre producción de guayaba puede resultar en una saturación del mercado, lo cual conllevaría a una rentabilidad más baja e incluso forzaría a ciertos productores a dejar la industria. Los productores de guayaba rosada controlan la mosca de la fruta con insecticidas. A pesar de esto, la amenaza que esta plaga representa protege a los productores locales de mercados extranjeros debido a lo estrictos requisitos de importación.

\section{Referencias}

Crane, J. y C. Balerdi. 2016. Guava growing in the Florida home landscape. HS-4. Gainesville: University of Florida Institute of Food and Agricultural Sciences. Disponible en: https://edis.ifas.ufl.edu/publication/MG045

FAOSTAT. 2016. Data. Crops and livestock products. http:// www.fao.org/faostat/en/\#data/QCL

Morton, J. 1987. Guava. https://www.hort.purdue.edu/ newcrop/morton/guava.html

Mossler, M., and J. Crane. 2009. Florida crop/pest management profile: Papaya. CIR1402. Gainesville: University of Florida Institute of Food and Agricultural Sciences.

Popenoe, W. 1920. Manual of Tropical and Subtropical Fruits. New York: MacMillan.

USDA-AMS. 2017. Terminal Market Price. https://www.marketnews.usda.gov/mnp/

fv-report-config-step1?type=termPrice 
Cuadro 1. Costo anual de producción de un acre de Guayaba en el sur de la Florida.

\begin{tabular}{|c|c|c|c|}
\hline Descripción & Cantidad & Valor por acre & Valor por libra \\
\hline & Libras & (\$/acre/año) & (\$/Libra) \\
\hline \multicolumn{4}{|c|}{ Ingresos } \\
\hline Producción comercializable (lbs/acre) & 8,000 & & \\
\hline Precio FOB en Homestead & & & 2.00 \\
\hline Ingreso total & & $16,000.00$ & \\
\hline \multicolumn{4}{|c|}{ Costos de producción } \\
\hline Riego & & 200.00 & \\
\hline Fertilizantes & & 550.00 & \\
\hline Herbicidas & & 450.00 & \\
\hline Insecticidas & & 450.00 & \\
\hline Fungicidas & & 400.00 & \\
\hline Mano de obra & & 650.00 & \\
\hline Interés sobre el capital (5\%) & & 135.00 & \\
\hline Costos totales de producción & & $2,835.00$ & 0.35 \\
\hline \multicolumn{4}{|c|}{ Costos Fijos } \\
\hline \multicolumn{4}{|l|}{ Gastos generales en efectivo: } \\
\hline Seguro & & 100.00 & \\
\hline Impuestos & & 100.00 & \\
\hline \multicolumn{4}{|l|}{ Gastos generales no en efectivo: } \\
\hline Alquiler de tierras & & 500.00 & \\
\hline Otros & & 600.00 & \\
\hline Costos fijos totales & & $1,300.00$ & 0.16 \\
\hline Costos totales precosecha & & $4,135.00$ & 0.52 \\
\hline \multicolumn{4}{|c|}{ Costos de cosecha y comercialización } \\
\hline Cosecha y costos de venta & & $2,800.00$ & 0.35 \\
\hline Costos totales de cosecha y comercialización & & $2,800.00$ & 0.35 \\
\hline Costos totales & & $6,935.00$ & 0.87 \\
\hline Margen Bruto & & $10,365.00$ & 1.30 \\
\hline Retorno neto estimado & & $9,065.00$ & 1.13 \\
\hline
\end{tabular}


Cuadro 2. Análisis de sensibilidad, margen bruto por acre.

\begin{tabular}{|c|c|c|c|c|c|c|}
\hline \multicolumn{2}{|c|}{$\begin{array}{l}\text { Producción } \\
\text { (libras/acre) }\end{array}$} & \multicolumn{5}{|c|}{$\begin{array}{c}\text { Precio de venta (Mayorista) } \\
\text { (dólares/libra) }\end{array}$} \\
\hline & & 1.80 & 1.90 & 2.00 & 2.10 & 2.20 \\
\hline & & $(-10 \%)$ & $(-5 \%)$ & (Base) & $(+5 \%)$ & $(+10 \%)$ \\
\hline 7,200 & $(-10 \%)$ & $7,325.00$ & $8,045.00$ & $8,765.00$ & $9,485.00$ & $10,205.00$ \\
\hline 7,600 & $(-5 \%)$ & $8,045.00$ & $8,805.00$ & $9,565.00$ & $10,325.00$ & $11,085.00$ \\
\hline 8,000 & (Base) & $8,765.00$ & $9,565.00$ & $10,365.00$ & $11,165.00$ & $11,965.00$ \\
\hline 8,400 & $(+5 \%)$ & $9,485.00$ & $10,325.00$ & $11,165.00$ & $12,005.00$ & $12,845.00$ \\
\hline 8,800 & $(+10 \%)$ & $10,205.00$ & $11,085.00$ & $11,965.00$ & $12,845.00$ & $13,725.00$ \\
\hline
\end{tabular}

Cuadro 3. Análisis de sensibilidad, retorno neto por acre.

\begin{tabular}{|c|c|c|c|c|c|c|}
\hline \multicolumn{2}{|c|}{$\begin{array}{l}\text { Producción } \\
\text { (libras/acre) }\end{array}$} & \multicolumn{5}{|c|}{$\begin{array}{c}\text { Precio de venta (Mayorista) } \\
\text { (dólares/libra) }\end{array}$} \\
\hline & & 1.80 & 1.90 & 2.00 & 2.10 & 2.20 \\
\hline & & $(-10 \%)$ & $(-5 \%)$ & (Base) & $(+5 \%)$ & $(+10 \%)$ \\
\hline 7,200 & $(-10 \%)$ & $6,025.00$ & $6,745.00$ & $7,465.00$ & $8,185.00$ & $8,905.00$ \\
\hline 7,600 & $(-5 \%)$ & $6,745.00$ & $7,505.00$ & $8,265.00$ & $9,025.00$ & $9,785.00$ \\
\hline 8,000 & (Base) & $7,465.00$ & $8,265.00$ & $9,065.00$ & $9,865.00$ & $10,665.00$ \\
\hline 8,400 & $(+5 \%)$ & $8,185.00$ & $9,025.00$ & $9,865.00$ & $10,705.00$ & $11,545.00$ \\
\hline 8,800 & $(+10 \%)$ & $8,905.00$ & $9,785.00$ & $10,665.00$ & $11,545.00$ & $12,425.00$ \\
\hline
\end{tabular}

